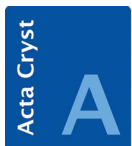

FOUNDATIONS

ADVANCES

ISSN 2053-2733

Keywords: multiple Bragg reflection; imperfect crystal

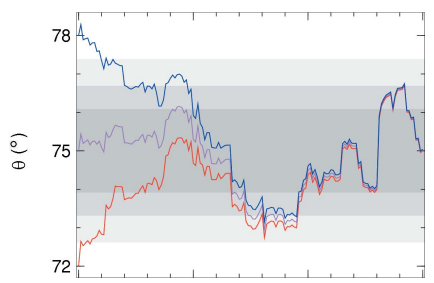

(C) 2020 International Union of Crystallography

\section{Multiple Bragg reflection by a thick mosaic crystal. Corrigendum}

\author{
Joachim Wuttke*
}

Jülich Centre for Neutron Science at MLZ, Forschungszentrum Jülich GmbH, 85747 Garching, Germany.

*Correspondence e-mail: j.wuttke@fz-juelich.de

An inconsistent approximation in Wuttke [Acta Cryst. (2014), A70, 429-440] is corrected. Section 3.5 on the polar angle random walk is withdrawn.

Section 3.5 of Wuttke (2014) investigates how multiple Bragg reflections change the polar angle of the radiation propagation vector. This entire section is withdrawn because of an error in the initial equation (64). Equation (64) was based on the series expansions (33) and (62), which were truncated at inconsistent orders. Since the $\alpha$ expansion (33) is used up to order $\beta^{2}$, a term in $\beta^{2}$ should also have been retained in (62). This leads to some cancellation and has the consequence that the term $\varepsilon_{j}^{2}$ in (64) should not be present. This invalidates the quantitative theory developed in Section 3.5. The restoring force that explains the confined random walk, observed in the Monte Carlo simulations and reported in the upper panel of Fig. 6, is of higher order in $\varepsilon$.

\section{References}

Wuttke, J. (2014). Acta Cryst. A70, 429-440. 